\title{
Synthesis and Biological Evaluation of Some Novel S- $\beta$-D-Glucosides of 4-Amino-5-alkyl-1,2,4-triazole-3-thiones Derivatives
}

\author{
Anila Rahimi Aghkand, ${ }^{1}$ Karim Akbari Dilmaghani, ${ }^{1, *}$ \\ Zahra Dono Ghezelbash ${ }^{1}$ and Behvar Asghari ${ }^{2}$ \\ ${ }^{1}$ Department of Organic Chemistry, Faculty of Chemistry, Urmia University, Urmia, 57159, Iran \\ ${ }^{2}$ Department of Horticultural Sciences Engineering, Faculty of Agriculture and Natural Resources, \\ Imam Khomeini International University, Qazvin, Iran \\ * Corresponding author: E-mail: k.adilmaghani@urmia.ac.ir \\ Tel: (+98)914-443-1392. Fax: (+98)44-357153-165
}

Received: 11-10-2018

\begin{abstract}
A novel series of 3-S- $\beta$-D-glucosides-4-arylideneamino-5-alkyl-1,2,4-triazoles were designed and synthesized by reaction of 4-amino-5-alkyl-4H-1,2,4triazole-3-thiol Schiff bases and 2,3,4,6-tetra-O-acetyl- $\alpha$-D-glucopyranosyl bromide. The structures of the target compounds have been characterized by ${ }^{1} \mathrm{H}$ NMR, ${ }^{13} \mathrm{C}$ NMR, FT-IR, and Microanalyses. All the newly synthesized compounds have been screened for their in vitro antibacterial and antifungal activities against two Gram-positive bacteria [Bacillus cereus (PTCC 1015) and Staphylococcus aureus (ATCC 25923)], two Gram-negative bacteria [Pseudomonas aeruginosa (ATCC 27853) and Escherichia coli (PTCC 1399) and two fungi [Aspergillus niger (PTCC 5012) and Candida albicans (PTCC 5027)].
\end{abstract}

Keywords: 4-amino-5-alkyl-4H-1,2,4-triazole-3-thiol; acetobromoglucose; thiocarbohydrazide; antimicrobial activity; antifungal activity; schiff base

\section{Introduction}

Antibiotics are drugs used for treating infection caused by microorganism such as bacteria or fungi and antibiotic resistance is the ability of microorganism to stand the effect of antibiotic. The resistance of infective bacteria to present antibiotics remains a clinical obstacle in the chemotrapy of many cancers to overcome the rapid development of drug resistance, new agents should preferably have chemical characteristics that clearly differ from those of existing agents. Thus led to the design and synthesize the new antimicrobial agents. 1,2,4-Triazole and its derivatives are an important class of compounds which possess diverse biological activities including anti-microbial, ${ }^{1,2}$ antibacterial, ${ }^{3,4}$ antifungal, ${ }^{5,6}$ anti-inflammatory, ${ }^{7}$ insecticidal, ${ }^{8}$ anticonvulsant, ${ }^{9,10}$ antitumor activity, ${ }^{11}$ anti HIV activity, ${ }^{12,13}$ hypoglycemic ${ }^{14}$ and anticonvulsant. ${ }^{15,16}$ Triadimefon and fluconazole which exhibits excellent fungicidal activities possessing 1,2,4-triazole nucleus. ${ }^{17,18}$
Compounds containing an azomethine group known as Schiff bases and are formed by the condensation of a primary amine with a carbonyl compound. Schiff bases attract much interest due to their synthetic availability along with antibacterial ${ }^{19-22}$ and antitumor ${ }^{23}$ properties.

The synthesis and investigation of biological activity of 1,2,4-triazole glycosides have been stimulated by the finding that Ribavirin, $\beta$-D-ribofuranosyl-1,2,4-triazole-3-carboxamide, as potent drug against DNA and RNA viruses. ${ }^{24}$

Moreover, sulfur-containing heterocycles represent an important group of sulfur compounds that are promising for use in practical applications. Therefore, it is interesting to report the synthesis of a new series of compounds in which the glycosyl moieties have been used as carriers for the heterocycles having the oxadiazole or triazole ring. It has been reported that the attachment of carbohydrate moieties to the 1,2,4-triazole nucleus through a thioglycosidic linkage enhances its antimicrobial activity. ${ }^{25}$

Some novel S- $\beta$-D-glucosides of 5 -aryl-1,2,4-triazole-3-thiones derivatives exhibited antibacterial and an- 
tifungal activities. ${ }^{26}$ In our previous work, ${ }^{27,28}$ we reported the synthesis and antibacterial properties of new series of thioglycoside derivatives of 1,2,4-triazole moieties.

The above facts and our interest on design of potent antibacterial agents with 1,3,4-oxadiazoles and 1,2,4-triazole moieties, promoted us to synthesis novel substituted thioglycosides by reaction of $\alpha-D$ acetobromoglucose (6) with 4-amino-5-alkyl-4H-1,2,4-triazole-3-thiol Schiff bases $\mathbf{5}(\mathbf{a}-\mathbf{g})$. The newly synthesized multicompounds $\mathbf{7}(\mathbf{a}-\mathbf{g})$ are useful in probing biological activity such as antibacterial and antifungal effects.

\section{1. Antimicrobial Activity}

\section{1. 1. Bacterial and Fungal Strains}

Two Gram-positive bacteria Bacillus cereus (PTCC 1015) and Staphylococcus aureus (ATCC 25923)], two Gram-negative bacteria [Pseudomonas aeruginosa (ATCC 27853) and Escherichia coli (PTCC 1399) and two fungi Aspergillus niger (PTCC 5012) and Candida albicans (PTCC 5027)] were used in antimicrobial assays. Microbial strains were obtained from the Pasteur Institute of Iran.

\section{2. Determination of Inhibition Zone}

The agar disc diffusion method was used for antimicrobial activity determination of the compounds using a previously described standard method. ${ }^{29} 100 \mu \mathrm{l}$ of the tested microorganisms suspensions, adjusted to 106-108 $\mathrm{CFU} / \mathrm{mL}$ were spread on the solid media plates. The compounds were dissolved in dimethyl sulphoxide and filtered by $0.45 \mu \mathrm{m}$ Millipore filters for sterilization. The paper discs (6 $\mathrm{mm}$ in diameter) werm saturated with $10 \mu \mathrm{l}$ of sample solution and placed on the inoculated agar. DMSO was used as the untreated control. These plates were incubated for $24 \mathrm{~h}$ at $37^{\circ} \mathrm{C}$ for bacterial strains and $48 \mathrm{~h}$ at $30^{\circ} \mathrm{C}$ for the yeasts. The diameter of inhibition zone (IZ) was measured in $\mathrm{mm}$ (including $6 \mathrm{~mm}$ diameter of paper disc). Gentamicin $(10 \mu \mathrm{g} /$ disc $)$ and nystatin (50 IU) were used as positive control in antibacterial and antifungal assays, respectively. Analyses were performed in triplicate and expressed as average values \pm SEM.

\section{3. Determination of Minimum Inhibitory Concentrations (MICs)}

The MIC values of the compounds were determined using the method of micro-well dilution assay. ${ }^{30-31}$ Briefly, the inoculants of the microbial strains were prepared from freshly cultured microorganisms that were adjusted to 0.5 McFarland standard turbidity. Serial dilutions of the compounds and standard samples were made in a concentration range from 5 to $1000 \mu \mathrm{g} / \mathrm{mL}$ in 96 -well plates, containing Mueller-Hinton broth for bacterial strains and Sabouraud dextrose broth for yeast. Gentamicin and nystatin were used as standard drugs in identical conditions to test materials. The plates were covered with sterile plate sealers and then incubated at $37^{\circ} \mathrm{C}$ under normal atmospheric condition for $24 \mathrm{~h}$ for bacterial strains and at $30^{\circ} \mathrm{C}$ for $48 \mathrm{~h}$ for yeasts. The MIC values were considered as the minimum concentration of the sample which could inhibit the growth of microorganisms.

\section{Result and Discussion}

4-amino-5-alkyl-4H-1,2,4-triazole-3-thioles 3(a-c) were prepared by the condensation of aliphatic carboxylic acids $2(\mathbf{a}-\mathbf{c})$ with thiocarbohydrazide. The reaction is improved by using carboxylic acids at thiocarbohydrazide melting point. This reaction is the selective method for the preparation of 4-amino-5-aryl/alkyl-4H-1,2,4-triazole-3thioles $^{32}$ (Scheme 1).<smiles>[R]C=Nn1c([R])n[nH]c1=S</smiles>

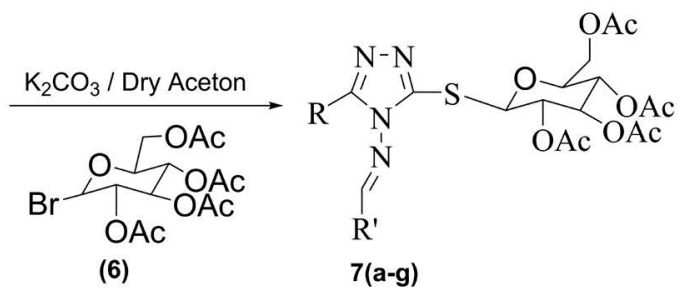

5a,7a: $R=M e(a), R^{\prime}=$

5b,7b: $R=M e(a), R^{\prime}=$

5c,7c: $R=M e(a), R^{\prime}=$

$5 d, 7 d: R=E t(b), R^{\prime}=$

5e,7e: $R=E t(b), R^{\prime}=$

5f,7f: $R=E t(b), \quad R^{\prime}=$

5g,7g: $R=\operatorname{Pr}(\mathbf{c}), \mathbf{R}^{\prime}=$
Pyridin-4-yl (d)

Pyridin-3-yl (e)

Pyridin-2-yl (f)

Pyridin-4-yl (d)

Pyridin-3-yl (e)

Pyridin-2-yl (f)

Pyridin-3-yl (e)

Scheme1. Synthesis of 3-S- $\beta$-D-glucosides-4-arylideneamino-5-alkyl-1,2,4-triazoles. 
Table1. Diameter of inhibition zone (IZ) values of the compounds against 2 gram (+) bacteria, 2 gram (-) bacteria and 2 fungi $(\mu \mathrm{g} / \mathrm{mL})$

\begin{tabular}{|c|c|c|c|c|c|c|c|c|c|c|c|c|c|c|c|c|}
\hline & $5 \mathbf{a}$ & $7 \mathbf{a}$ & $5 \mathbf{b}$ & $7 \mathbf{b}$ & $5 c$ & $7 \mathrm{c}$ & $5 d$ & $7 d$ & $5 e$ & $7 e$ & $5 f$ & $7 f$ & $5 \mathrm{~g}$ & $7 \mathrm{~g}$ & Gentamicin & Nystatin \\
\hline $\begin{array}{l}\text { Escherichia coli } \\
\operatorname{gram}(-)\end{array}$ & - & 6.7 & - & 6.3 & - & 6.7 & - & - & - & 6.1 & - & - & - & 7.2 & 23.4 & - \\
\hline $\begin{array}{l}\text { Pseudomonas } \\
\text { aeruginosa } \\
\operatorname{gram}(-)\end{array}$ & - & 6.6 & - & - & - & 6.5 & - & 6.5 & - & - & - & - & - & 6.7 & 27.3 & - \\
\hline $\begin{array}{l}\text { Staphylococcus } \\
\text { aureus } \\
\operatorname{gram}(+)\end{array}$ & 6.7 & 12.4 & 6.3 & 14.3 & 6.4 & 11.4 & - & 13.1 & 6.5 & 9.1 & - & 8.4 & - & 9.3 & 28.5 & - \\
\hline $\begin{array}{l}\text { Bacillus cereus } \\
\operatorname{gram}(+) \\
\text { Aspergillus }\end{array}$ & 6.4 & 14.7 & 7.1 & 15.6 & 6.5 & 9.8 & 6.2 & 13.9 & 6.6 & 8.2 & - & 10.7 & - & 9.5 & 31.6 & - \\
\hline $\begin{array}{l}\text { niger } \\
\text { fungi }\end{array}$ & 7.1 & 15.7 & 8.7 & 13.4 & 7.5 & 11.2 & 6.4 & 10.2 & 8.2 & 12.1 & 7.3 & 10.1 & 6.8 & 9.7 & - & 22.6 \\
\hline $\begin{array}{l}\text { Candida } \\
\text { albicans } \\
\text { fungi }\end{array}$ & 6.3 & 17.5 & 7.9 & 14.1 & 7.3 & 10.1 & 6.3 & 14.2 & 8.7 & 12.4 & 6.6 & 12.7 & 6.4 & 11.3 & - & 26.8 \\
\hline
\end{tabular}

Schiff bases $\mathbf{5}(\mathbf{a}-\mathbf{g})$ have been synthesized by a reaction of 3-alkyl-4-amino-1,2,4-triazole-5-thione $\mathbf{3}(\mathbf{a}-\mathbf{c})$ with pyridine aldehydes $\mathbf{4}(\mathbf{d}-\mathbf{f})$ in absolute ethanol as solvent in presence of glacial acetic acid as a catalyst. The IR spectra of the compounds $\mathbf{3}(\mathbf{a}-\mathbf{c})$ showed the absorptions at $3100,1330 \mathrm{~cm}^{-1}$ which were attributed to $\mathrm{N}-\mathrm{H}$ and $\mathrm{C}=\mathrm{S}$ stretching vibration and a strong absorption at $1580 \mathrm{~cm}^{-1}$ which was assigned to the $\mathrm{C}=\mathrm{N}$ stretching vibration. The ${ }^{1} \mathrm{H}$ NMR spectra showed a singlet signal at about $10 \mathrm{ppm}$ due to $(\mathrm{CH}=\mathrm{N})$ and the absence of the chemical shift of $4-\mathrm{NH}_{2}$ in the spectra of $\mathbf{3}(\mathbf{a}-\mathbf{c})$ proving that the title Schiff bases $\mathbf{5}(\mathbf{a}-\mathbf{g})$ were formed.

In our attempt to obtain $\alpha$-acetobromoglucose (6) at the first step D-glucose were treated with acetic anhydride in pyridine at room temperature gave $1,2,3,4,6$-penta- $O$ acetyl- $\beta$-glucose and the anomeric bromination of this compound with hydrogen bromide in acetic acid gave 2,3,4,6-tetra- $O$-acetyl- $\alpha$-glucopyranosyl bromide (6).

The existence of thiol-thione tautomerism is known for the compounds $\mathbf{3}(\mathbf{a}-\mathbf{c})$ and generally one forms is predominant. $^{34-37}$

3-S- $\beta$-D-glucosides-4-arylideneamino-5-alkyl-1,2,4 -triazoles $7(\mathbf{a}-\mathbf{g})$ were synthesized by the reaction of 3 -alkyl-4-amino-1,2,4-triazole-5-thione Schiff bases $\mathbf{5}(\mathbf{a}-\mathbf{g})$ and the peracetylated $\beta$-pyranosyl bromide (6) in the presence of potassium carbonate as a weak base in dry acetone. (Scheme 1).

Anomeric $\beta$-configurations of the $S$-linked glycosides $7(\mathbf{a}-\mathbf{g})$ were supported by their ${ }^{1} \mathrm{H}$ NMR data. The chemical shifts of the anomeric proton signals of thioglycosides revealed around $\delta(6.20)$ with a large coupling constant $J_{1,2}$ values of (9.3) $\mathrm{Hz}$ which consistent with the reported data for $\mathrm{S}-\beta-\mathrm{D}$ glycosides.

The result of the antibacterial and antifungal activity shows that all the compounds have lesser activity than corresponding standard compounds and the target com- pounds exhibited better antifungal activity than anti-bacterial activity. However the anti-bacterial results showed that against Staphylococcus aureus compounds (7b) and (7d); against Bacillus cereus compounds (7b), (7a) and (7d); have comparable activity with Gentamicin as Standard. The antifungal study results revealed that against $A s$ pergillus niger compounds (7a)

(7b) and (7e); against Candida albicans (7a), (7b) and (7d) have comparable activity with Nystatin as standard. Though compound (7b) was found to have the highest activity against Bacillus cereus and compound (7a) was found to have the highest activity against Candida albicans among all the tested compounds.

\section{Experimental}

\section{1. General}

The melting points of all compounds were recorded on a Philip Harris C4954718 apparatus without calibration.IR and ${ }^{1} \mathrm{H}$ - and ${ }^{13} \mathrm{C}-\mathrm{NMR}$ spectra were recorded on Thermo Nicolet Nexus 670 FT-IR and Bruker Avance 300 $\mathrm{MHz}$ spectrometers, respectively. Thin layer chromatography (TLC) analyses were carried out on silica gel plates. All chemicals were purchased from Merck (Tehran, Iran) and used as received by standard procedures. All of the instruments, chemicals and solvents were dried according to standard methods. Freshly distilled solvents were used throughout, and anhydrous solvents were dried according to the method reported by Perrin and Armarego. ${ }^{38}$ Microanalyses were performed on a Leco Analyzer 932.

\section{2. General Procedure of Synthesis of 4-amino-5-alkyl-4H-1,2,4-triazole-3- thiol 3(a-c):}


A mixture of thiocarbohydrazide $(0.01 \mathrm{~mol})$ and carboxylic acid $(0.01 \mathrm{~mol})$ was heated until it melted. The mixture was consistently refluxed for $40 \mathrm{~min}$. The product obtained on cooling was treated with a sodium bicarbonate solution to neutralize the unreacted acid if any. The product was then washed with water and collected by filtration. The solid product was recrystallized from a distilled water. ${ }^{32}$

General Procedure for Synthesis of Schiff bases of (E) -5-alkyl-4-((pyridin-3-ylmethylene) amino)-2,4-dihydro-3H-1,2,4-triazole-3-thione 5(a-g):

To a suspension of substituted pyridine carboxaldehyde $4(\mathbf{d}-\mathbf{f})(0.2 \mathrm{~mol})$ in ethanol $(1 \mathrm{~mL})$, an equimolar amount of corresponding 4-amino-5-alkyl-4H-1,2,4-triazole-3-thiol $(0.1 \mathrm{~mol}) \mathbf{3}(\mathbf{a}-\mathbf{c})$ was added. The suspension was heated until a clear solution was obtained. Then few drops of concentrated sulfuric acid were added, and the solution was heated for $6 \mathrm{hrs}$ on a water bath, the precipitate solid was filtered off and recrystallized from in ethanol. ${ }^{33}$

General Procedure for Synthesis of 2,3,4,6-tetra-O-acetyl-a-D-glucopyranosyl bromide (6):

It was also prepared according to the literature procedure. $^{39}$

General Procedure for Synthesis of 3-S- $\beta$-D-glucosides-4-arylideneamino-5-alkyl-1,2,4-triazoles $(7 \mathrm{a}-\mathrm{g})$ :

A mixture of compound $\mathbf{5}(\mathbf{a}-\mathbf{g})(1 \mathrm{mmol})$ and potassium carbonate $(1 \mathrm{mmol}, 0.138 \mathrm{~g})$ in dry acetone $(25 \mathrm{~mL})$ and 2 drops of Dimethylformamide were stirred for $1 \mathrm{hrs,}$ then glycosyl bromide (6) (1.2 mmol, $0.410 \mathrm{~g})$ was added. Stirring was continued overnight, and then the reaction mixture was heated under reflux for 2-4 hrs. After cooling, the mixture was filtered, then the precipitate of the compound was submitted to column chromatography $\left(\mathrm{SiO}_{2}\right.$, EtOAc/hexane, 3:8). ${ }^{27}$

(E)-5-methyl-4-((pyridin-4-ylmethylene) amino)-2,4dihydro-3H-1,2,4-triazole-3-thione (5a):

(Yellow crystals), (Yield\% = 74) (0.32 g) m.p: $267-$ $269^{\circ} \mathrm{C}$, FT-IR $\left(\mathrm{KBr}, \mathrm{v} \mathrm{cm}{ }^{-1}\right): 3042(\mathrm{C}-\mathrm{H}, \mathrm{Ar}), 2839\left(\mathrm{CH}_{3}\right)$, $1592(\mathrm{C}=\mathrm{N}), 1278(\mathrm{C}=\mathrm{S}) \mathrm{cm}^{-1},{ }^{1} \mathrm{H}$ NMR $(300 \mathrm{MHz}$, DMSO): $\delta$ (ppm), $2.36\left(\mathrm{~s}, 3 \mathrm{H}, \mathrm{CH}_{3}\right), 7.82(\mathrm{~d}, J=5.1 \mathrm{~Hz}, 2 \mathrm{H}$ Pyridin) 8.75 (d, J=5.1Hz, 2H Pyridin) $10.32(\mathrm{~s}, 1 \mathrm{H}$, $\mathrm{HC}=\mathrm{N}) 13.75(\mathrm{~s}, 1 \mathrm{H}, \mathrm{NH}) .{ }^{13} \mathrm{C}-\mathrm{NMR}\left(75 \mathrm{MHz}, \mathrm{CDCl}_{3}\right): \delta$ (ppm), 11.15, 122.32, 139.97, 149.27, 151.38, 161.78, 162.33

(E)-5-methyl-4-((pyridine-3-ylmethylene) amino)-2,4dihydro-3H-1,2,4-triazole-3-thione (5b):

(Light Yellow crystals) (Yield\% $=76)(0.83$ gr), m.p: 239-241 ${ }^{\circ} \mathrm{C}$, FT-IR ( $\mathrm{KBr}, \mathrm{v} \mathrm{cm}{ }^{-1}: 3061$ (C-H Ar), $2852\left(\mathrm{CH}_{3}\right)$, $1590(\mathrm{C}=\mathrm{N}), 1274(\mathrm{C}=\mathrm{S}) \mathrm{cm}^{-1},{ }^{1} \mathrm{H}$ NMR (300 MHz, DMSO): $\delta(\mathrm{ppm}), 2.35\left(\mathrm{~s}, 3 \mathrm{H}, \mathrm{CH}_{3}\right), 7.53-7.57$ ( $\mathrm{m}, 1 \mathrm{H}$, pyridine), 8.29 (d, $J=7.8 \mathrm{~Hz}, 1 \mathrm{H}$ pyridine) 8.73 (d, $J=4.5 \mathrm{~Hz}, 1 \mathrm{H}$ pyridine) 9 (s, $1 \mathrm{H}$, pyridine) $10.19(\mathrm{~s}, 1 \mathrm{H}, \mathrm{HC}=\mathrm{N}) 13.79(\mathrm{~s}, 1 \mathrm{H}, \mathrm{NH})$. ${ }^{13} \mathrm{C}-\mathrm{NMR}\left(75 \mathrm{MHz}, \mathrm{CDCl}_{3}\right): \delta(\mathrm{ppm}), 11.17,124.71,128.75$, 135.34, 135.40, 149.05, 150.38, 153.36, 161.63.

(E)-5-methyl-4-((pyridin-2-ylmethylene) amino)-2,4dihydro-3H-1,2,4-triazole-3-thione) (5c):

(Pale green crystals), m.p :194-196 ${ }^{\circ} \mathrm{C}($ Yield $\%=82)$ $(0.18 \mathrm{~g})$ FT-IR $\left(\mathrm{KBr}, \mathrm{v} \mathrm{cm}{ }^{-1}\right): 3104(\mathrm{NH}), 3066(\mathrm{C}-\mathrm{H} \mathrm{Ar})$ $2934\left(\mathrm{CH}_{3}\right), 1586(\mathrm{C}=\mathrm{N}), 1287(\mathrm{C}=\mathrm{S}) \mathrm{cm}^{-1},{ }^{1} \mathrm{H}$ NMR $(300$ $\mathrm{MHz}, \mathrm{DMSO}): \delta(\mathrm{ppm}), 2.37\left(\mathrm{~s}, 3 \mathrm{H}, \mathrm{CH}_{3}\right) 7.56$ (t, $J=3.9$, $1 \mathrm{H}$, pyridine) $7.83(\mathrm{t}, J=7.5,1 \mathrm{H}$, pyridine $) 8.14(\mathrm{~d}, J=5.4$ $1 \mathrm{H}$, Pyridine $) 8.25(\mathrm{~d}, J=4.8,1 \mathrm{H}$, Pyridin $) 10.35(\mathrm{~s}, 1 \mathrm{H}$, $\mathrm{HC}=\mathrm{N}) 13.79(\mathrm{~s}, 1 \mathrm{H}, \mathrm{NH}) .{ }^{13} \mathrm{C}-\mathrm{NMR}\left(75 \mathrm{MHz}, \mathrm{CDCl}_{3}\right): \delta$ $(\mathrm{ppm}), 10.87,120.66,127.64,136.54,138.66,149.20$, $151.44,160.23,161.74$.

(E)-5-ethyl-4-((pyridin-4-ylmethylene) amino)-2,4-dihydro-3H-1,2,4-triazole-3-thione) (5d):

(Yellow crystals), (Yield\% = 73) (0.17 g) m.p: 246$248^{\circ} \mathrm{C}$, FT-IR $\left(\mathrm{KBr}, \mathrm{v} \mathrm{cm} \mathrm{cm}^{-1}\right): 3041$ (C-H Ar), 2973 (Et), $1591(\mathrm{C}=\mathrm{N}), 1268(\mathrm{C}=\mathrm{S}) \mathrm{cm}^{-1} \cdot{ }^{1} \mathrm{H}$ NMR $(300 \mathrm{MHz}$, DMSO): $\delta$ (ppm), $1.22\left(\mathrm{t}, J=7.2,3 \mathrm{H}, \mathrm{CH}_{3}\right), 2.73-2.81(\mathrm{~m}$, $\left.2 \mathrm{H}, \mathrm{CH}_{2}\right) 7.82(\mathrm{~d}, J=4.5 \mathrm{~Hz}, 2 \mathrm{H}$ pyridine) $8.76(\mathrm{~d}, J=4.5$ $\mathrm{Hz}, 2 \mathrm{H}$ pyridine $) 10.34(\mathrm{~s}, 1 \mathrm{H}, \mathrm{HC}=\mathrm{N}) 13.85(\mathrm{~s}, 1 \mathrm{H}$, $\mathrm{NH}) .{ }^{13} \mathrm{C}-\mathrm{NMR}\left(75 \mathrm{MHz}, \mathrm{CDCl}_{3}\right): \delta(\mathrm{ppm}), 11.34,18.65$, $121.23,140.03,150.21,153.14,160.71,161.93$.

(5e): ( (E)-5-ethyl-4-((pyridin-3-ylmethylene) amino)2,4-dihydro-3H-1,2,4-triazole-3-thione

(Yellow crystals), m.p:188-190 ${ }^{\circ} \mathrm{C},($ Yield\% $=72)$ (0.08 g), FT-IR $\left(\mathrm{KBr}, \mathrm{v} \mathrm{cm}^{-1}\right): 3097(\mathrm{NH}), 3048(\mathrm{CH}-\mathrm{Ar})$, $2975(\mathrm{Et}), 1583(\mathrm{C}=\mathrm{N}), 1278(\mathrm{C}=\mathrm{S}) \mathrm{cm}^{-1} .{ }^{1} \mathrm{H}$ NMR $(300$ $\mathrm{MHz}, \mathrm{DMSO}): \delta(\mathrm{ppm}), 1.22\left(\mathrm{t}, J=7.2,3 \mathrm{H}, \mathrm{CH}_{3}\right), 2.72-2.80$ $\left(\mathrm{m}, 2 \mathrm{H}, \mathrm{CH}_{2}\right) 7.56-7.60(\mathrm{~m}, 1 \mathrm{H}$, pyridine), 8.31 (d, $J=6.6$ $\mathrm{Hz}, 1 \mathrm{H}$ Pyridine) $8.76(\mathrm{~d}, J=4.5 \mathrm{~Hz}, 1 \mathrm{H}$ pyridine) $9.02(\mathrm{~s}$, $1 \mathrm{H}$, pyridine) $10.19(\mathrm{~s}, 1 \mathrm{H}, \mathrm{HC}=\mathrm{N}) 14.81(\mathrm{~s}, 1 \mathrm{H}, \mathrm{NH})$. ${ }^{13} \mathrm{C}-\mathrm{NMR}\left(75 \mathrm{MHz}, \mathrm{CDCl}_{3}\right): \delta(\mathrm{ppm}), 9.73,18.66,125.79$, $128.78,134.38,136.47,151.58,152.01,159.53,161.82$.

(E)-5-ethyl-4-((pyridin-2-ylmethylene) amino)-2,4-dihydro-3H-1,2,4-triazole-3-thione (5f):

(Brown crystals), m.p:168-170 ${ }^{\circ} \mathrm{C}$, (Yield\% $=86$ ) $(0.2 \mathrm{~g})$, FT-IR $\left(\mathrm{KBr}, \mathrm{v} \mathrm{cm}^{-1}\right)$ : $3119(\mathrm{NH}), 3068(\mathrm{C}-\mathrm{H} \mathrm{Ar})$, 2918 (Et), $1579(\mathrm{C}=\mathrm{N}), 1285(\mathrm{C}=\mathrm{S}) \mathrm{cm}^{-1} \cdot{ }^{1} \mathrm{H}$ NMR $(300$ $\mathrm{MHz}, \mathrm{DMSO}): \delta(\mathrm{ppm}), 1.23\left(\mathrm{t}, J=7.5,3 \mathrm{H}, \mathrm{CH}_{3}\right) 2.74-2.81$ $\left(\mathrm{m}, 2 \mathrm{H}, \mathrm{CH}_{2}\right) 7.56(\mathrm{t}, J=6.6,1 \mathrm{H}$, pyridine) $7.97(\mathrm{t}, J=6.9$, $1 \mathrm{H}$, pyridine) $8.13(\mathrm{~d}, J=8.1,1 \mathrm{H}$, Pyridin $) 8.73(\mathrm{~d}, J=4.2$, $1 \mathrm{H}$, Pyridin) $10.35(\mathrm{~s}, 1 \mathrm{H}, \mathrm{HC}=\mathrm{N}) 13.83(\mathrm{~s}, 1 \mathrm{H}, \mathrm{NH})$. ${ }^{13} \mathrm{C}-\mathrm{NMR}\left(75 \mathrm{MHz}, \mathrm{CDCl}_{3}\right): \delta(\mathrm{ppm}), 11.33,18.71,120.71$, $122.88,127.77,136.74,138.88,149.57,159.87,162.02$.

(E)-5-propyl-4-((pyridin-3-ylmethylene) amino)-2,4dihydro-3H-1,2,4-triazole-3-thione (5g):

(Yellow crystals), (Yield\% $=77$ ) (0.38 gr) m.p:166$168^{\circ} \mathrm{C}$, FT-IR $\left(\mathrm{KBr}, \mathrm{v} \mathrm{cm}^{-1}\right): 3057(\mathrm{CH}-\mathrm{Ar}), 1586(\mathrm{C}=\mathrm{N})$, 
$1278(\mathrm{C}=\mathrm{S}) \mathrm{cm}^{-1} \cdot{ }^{1} \mathrm{H}$ NMR (300 MHz, DMSO): $\delta$ (ppm), $0.94\left(\mathrm{t}, J=7.5,3 \mathrm{H}, \mathrm{CH}_{3}\right), 1.63-1.72\left(\mathrm{~m}, 2 \mathrm{H}, \mathrm{CH}_{2}\right) 2.73(\mathrm{t}$, $\left.J=7.5,2 \mathrm{H}, \mathrm{CH}_{2}\right) 7.56-7.60(\mathrm{~m}, 1 \mathrm{H}$, pyridine $), 8.31(\mathrm{~d}, J=6.3$ $\mathrm{Hz}, 1 \mathrm{H}$ pyridine) $8.76(\mathrm{~d}, J=4.8 \mathrm{~Hz}, 1 \mathrm{H}$ pyridine) $9.02(\mathrm{~s}$, $1 \mathrm{H}$, pyridine) $10.19(\mathrm{~s}, 1 \mathrm{H}, \mathrm{HC}=\mathrm{N}) 13.81(\mathrm{~s}, 1 \mathrm{H}, \mathrm{NH})$. ${ }^{13} \mathrm{C}-\mathrm{NMR}\left(75 \mathrm{MHz}, \mathrm{CDCl}_{3}\right): \delta$ (ppm), 13.87, 19.34, 26.75, $124.79,128.78,135.40,151.72,153.44,159.66,161.85$, 162.33 .

Synthesis of 1,2,3,4,6-penta-O-acetyl-a-D-glucopyranose: It was also prepared according to the literature procedure. ${ }^{36}$ (Yield\% $\left.=68\right)(7.5 \mathrm{gr})$ (White precipitate), FT-IR $\left(\mathrm{KBr}, v \mathrm{~cm}^{-1}\right): 1748,1374,1227,{ }^{1} \mathrm{H}$ NMR $(300 \mathrm{MHz}$, $\mathrm{CDCl}_{3}$ ), $\delta: 2.01$ (s, 3H, OAc), 2.03 (s, 3H, OAc), 2.08 (s, $3 \mathrm{H}, \mathrm{OAc}), 2.11$ (s, 3H, OAc), 2.18 (s, 3H, OAc), 3.82-3.85 (m, 1H, H-5), 4.08-4.12 (m, 1H, H-6a), 4.26-4.32 (m, $1 \mathrm{H}$, H-6b), 5.09-5.28 (m, 3H, H-2, H-4, H-3), 5.71 (d, 1H, J1,2 $=8.4, \mathrm{H}-1) \cdot{ }^{13} \mathrm{C} \mathrm{NMR}\left(75 \mathrm{MHz}, \mathrm{CDCl}_{3}\right), \delta: 20.55(3 \mathrm{C})$, 20.69, $20.80\left(5 \times \mathrm{OCOCH}_{3}\right), 61.41(\mathrm{C}-6), 67.70(\mathrm{C}-4)$, 70.18 (C-2), 72.69 (C-3), 72.75 (C-5), 91.66 (C-1), 168.94, $169.23,169.37,170.08,170.59\left(5 \times \mathrm{OCOCH}_{3}\right)$.

Synthesis of 2,3,4,6-tetra-O-acetyl-a-D-glucopyranosyl bromide (6): It was also prepared according to the literature procedure. ${ }^{39}$ White precipitate; $57 \%$ (1.2 g); FT-IR $\left(\mathrm{KBr}, v \mathrm{~cm}^{-1}\right): 1745,1377,1236,607 .{ }^{1} \mathrm{H}$ NMR $(300 \mathrm{MHz}$, $\mathrm{CDCl}_{3}$ ), $\delta: 2.03$ (s, 3H, OAc), 2.05 (s, 3H, OAc), 2.10 (s, 6H, OAc), $4.12(\mathrm{~d}, 1 \mathrm{H}, \mathrm{H}-6 \mathrm{a}), 4.28-4.36(\mathrm{~m}, 2 \mathrm{H}, \mathrm{H}-6 \mathrm{~b}$, H-5), $4.81-4.86\left(\mathrm{dd}, 1 \mathrm{H}, J_{1,2}=3.9, J_{2,3}=9.9, \mathrm{H}-2\right), 5.16$ $(\mathrm{t}, 1 \mathrm{H}, \mathrm{H}-4), 5.56(\mathrm{t}, 1 \mathrm{H}, \mathrm{H}-3), 6.61\left(\mathrm{~d}, 1 \mathrm{H}, J_{1,2}=3.9, \mathrm{H}-1\right)$. ${ }^{13} \mathrm{C}$ NMR (75 MHz, CDCl 3 ), $\delta: 20.53,20.60$ (2C), 20.63 (4 $\left.\times \mathrm{OCO}^{-} \mathrm{H}_{3}\right), 60.93(\mathrm{C}-6), 67.15(\mathrm{C}-4), 70.14(\mathrm{C}-2), 70.58$ (C-3), 72.12 (C-5), 86.54 (C-1), 169.44, 169.77, 169.82, $170.48\left(4 \times \mathrm{OCOCH}_{3}\right)$.

Synthesis of 4-(pyridine-4-yl methylene-amino) -5-methyl-2-yl-3- (2,3,4,6-tetra-O- acetyl- $\beta$-D-glucopyranosyl Sulfonyl) -1,2,4-triazole (7a): yellow crystals $76 \%$ (1.24 g), m.p : $74-76^{\circ} \mathrm{C}$; FT-IR $\left(\mathrm{KBr}, \mathrm{v} \mathrm{cm}^{-1}\right): 2947$ (C-H), $1753(\mathrm{C}=\mathrm{O}), 1601(\mathrm{HC}=\mathrm{N}), 1371\left(\mathrm{CH}_{3}\right), 1047,1230$ (C-O), ${ }^{1} \mathrm{H}$ NMR $\left(300 \mathrm{MHz}, \mathrm{CDCl}_{3}\right): \delta 1.94$ (s, $\left.3 \mathrm{H}, \mathrm{OAc}\right)$, 2.04 (s, 3H, OAc), 2.07 (s, 3H, OAc), 2.09 (s, 3H, OAc), 2.49 (s, 3H, $\left.\mathrm{CH}_{3}\right), 3.97-4(\mathrm{~m}, 1 \mathrm{H}, \mathrm{H}-6 \mathrm{a}), 4.15$ (d, $J=12.3$ $\mathrm{Hz}, 1 \mathrm{H}, \mathrm{H}-6 \mathrm{~b}), 4.28-4.34(\mathrm{~m}, 1 \mathrm{H}, \mathrm{H}-5), 5.25(\mathrm{t}, J=9.9 \mathrm{~Hz}$, $1 \mathrm{H}, \mathrm{H}-4), 5.42(\mathrm{t}, J=9.3 \mathrm{~Hz}, 1 \mathrm{H}, \mathrm{H}-2), 5.74(\mathrm{t}, J=9.3 \mathrm{~Hz}, 1 \mathrm{H}$, $\mathrm{H}-3$ ), 6.21 (d, $J=9.3 \mathrm{~Hz}, 1 \mathrm{H}, \mathrm{H}-1), 7.70$ (d, $J=4.8 \mathrm{~Hz}, 2 \mathrm{H}$ pyridine) $8.78(\mathrm{~d}, J=4.5 \mathrm{~Hz}, 2 \mathrm{H}$ pyridine) $10.83(\mathrm{~s}, 1 \mathrm{H}$, $\mathrm{HC}=\mathrm{N}) .{ }^{13} \mathrm{C}-\mathrm{NMR}\left(75 \mathrm{MHz}, \mathrm{CDCl}_{3}\right): \delta(\mathrm{PPM}), 11.22$, $20.57,61.67,67.73,69.14,73.61,74.59,81.28,121.85$, $139.95,149.30,150.65,162.33,164.04,168.89,169.35$, 170.15, 170.61. Calcd: C, 50.27; H, 4.95; N, 12.74; S, 5.83\%; Found: C, 50.37; H, 4.85; N, 12.94; S, 5.92\%.

Synthesis of 4-(pyridine-3-yl methylene-amino) -5-methyl-2-yl-3- $\quad(2,3,4,6$-tetra-O-acetyl- $\beta$-D-gluco pyranosyl Sulfonyl) -1,2,4-triazole (7b):
White crystals $73 \%\left(0.8\right.$ g), m.p: $68-70{ }^{\circ} \mathrm{C}$; FT-IR $\left(\mathrm{KBr}, \mathrm{v} \mathrm{cm}^{-1}\right): 2958(\mathrm{C}-\mathrm{H}), 1752(\mathrm{C}=\mathrm{O}), 1599(\mathrm{HC}=\mathrm{N})$, $1370\left(\mathrm{CH}_{3}\right), 1046,1231$ (C-O). ${ }^{1} \mathrm{H}$ NMR $(300 \mathrm{MHz}$, $\mathrm{CDCl}_{3}$ ): $\delta 1.93$ (s, 3H, OAc), 2.02 (s, 3H, OAc), 2.05 (s, 3H, OAc), 2.07 (s, 3H, OAc), $2.46\left(\mathrm{~s}, 3 \mathrm{H}, \mathrm{CH}_{3}\right), 3.96-4(\mathrm{~m}, 1 \mathrm{H}$, H-6a), 4.06-4.16 (m, 1H, H-6b), 4.27-4.32 (m, 1H, H-5), $5.24(\mathrm{t}, J=9.9 \mathrm{~Hz}, 1 \mathrm{H}, \mathrm{H}-4), 5.40(\mathrm{t}, J=9.6 \mathrm{~Hz}, 1 \mathrm{H}, \mathrm{H}-2)$, $5.72(\mathrm{t}, J=9.3 \mathrm{~Hz}, 1 \mathrm{H}, \mathrm{H}-3), 6.20(\mathrm{~d}, J=9.3 \mathrm{~Hz}, 1 \mathrm{H}, \mathrm{H}-1)$, 7.40-7.44 (m, 1H, pyridine), 8.19 (d, $J=7.8 \mathrm{~Hz}, 1 \mathrm{H}$ pyridine) $8.74(\mathrm{~d}, J=3.9 \mathrm{~Hz}, 1 \mathrm{H}$ pyridine $) 8.99(\mathrm{~s}, 1 \mathrm{H}$, pyridine $)$ $10.68(\mathrm{~s}, 1 \mathrm{H}, \mathrm{HC}=\mathrm{N}) .{ }^{13} \mathrm{C}-\mathrm{NMR}\left(75 \mathrm{MHz}, \mathrm{CDCl}_{3}\right): \delta$ (PPM), 11.27, 20.60, 61.64, 67.66, 69.10, 73.58, 74.50, $81.29,123.92,128.63,134.80,149.22,152.94,157.59$, 163.90, 168.94, 169.38, 170.17, 170.65. Calcd: C, 50.27; H, 4.95; N, 12.74; S, $5.83 \%$; Found: C, 50.17; H, 4.82; N, 12.86; S, $5.98 \%$.

(Synthesis of 4-(pyridine-2-yl methylene-amino) -5-methyl-2-yl-3- $\quad(2,3,4,6$-tetra-O-acetyl- $\beta$-D-gluco pyranosyl Sulfonyl) -1,2,4-triazole (7c):

White crystals $76 \%$ (1.24 g), m.p: $105-107^{\circ} \mathrm{C}$; FT-IR $\left(\mathrm{KBr}, \mathrm{cm}^{-1}\right): 2948(\mathrm{C}-\mathrm{HAr}), 1752(\mathrm{C}=\mathrm{O}), 1590(\mathrm{HC}=\mathrm{N})$, $1373\left(\mathrm{CH}_{3}\right), 1045,1234(\mathrm{C}-\mathrm{O}),\left(\mathrm{C}^{-1} \mathrm{H}\right.$ NMR $(300 \mathrm{MHz}$, $\left.\mathrm{CDCl}_{3}\right): \delta 1.94(\mathrm{~s}, 3 \mathrm{H}, \mathrm{OAc}), 2.03(\mathrm{~s}, 3 \mathrm{H}, \mathrm{OAc}), 2.06(\mathrm{~s}, 3 \mathrm{H}$, OAc), 2.09 (s, 3H, OAc), $2.48\left(\mathrm{~s}, 3 \mathrm{H}, \mathrm{CH}_{3}\right) 3.96-4.01$ (m, 1H, H-6a), 4.12 (d, J=7.2 Hz, 1H, H-6b), 4.28-4.34 (m, $1 \mathrm{H}, \mathrm{H}-5), 5.25(\mathrm{t}, J=9.9 \mathrm{~Hz}, 1 \mathrm{H}, \mathrm{H}-4), 5.41(\mathrm{t}, J=9.3 \mathrm{~Hz}, 1 \mathrm{H}$, $\mathrm{H}-2), 5.73(\mathrm{t}, J=9.3 \mathrm{~Hz}, 1 \mathrm{H}, \mathrm{H}-3), 6.23(\mathrm{~d}, J=9.3 \mathrm{~Hz}, 1 \mathrm{H}$, $\mathrm{H}-1), 7.40-7.44(\mathrm{~m}, 1 \mathrm{H}$, Pyridin) $7.83(\mathrm{t}, J=7.5 \mathrm{~Hz}, 1 \mathrm{H}$, Pyridin) 8.09 (d, $J=7.8 \mathrm{~Hz}, 1 \mathrm{H}$, Pyridin) $8.75(\mathrm{~d}, J=4.8 \mathrm{~Hz}$, $1 \mathrm{H}$, Pyridin), 10.63 (s, $1 \mathrm{H}, \mathrm{CH}=\mathrm{N}) .{ }^{13} \mathrm{C}-\mathrm{NMR}(75 \mathrm{MHz}$, $\left.\mathrm{CDCl}_{3}\right): \delta(\mathrm{PPM}), 11.26,20.58,61.70,67.75,69.20,73.59$, 74.52, 81.37, 121.96, 125.91, 136.74, 149.13, 150.15, 151.92, 162.33, 164.12, 168.97, 169.39, 170.13, 170.65. Calcd: C, 50.27; H, 4.95; N, 12.74; S, 5.83\%; Found: C, 50.17; H, 4.82; N, 12.86; S, 5.98\%.

Synthesis of 4-(pyridine-4-yl methylene-amino) -5-ethyl-2-yl-3- $(2,3,4,6$-tetra-O-acetyl- $\beta$-D-gluco pyranosyl Sulfonyl) -1,2,4-triazole (7d):

Orange crystals $77 \%(0.085 \mathrm{~g})$, m.p: $59-61{ }^{\circ} \mathrm{C}$; FT-IR (KBr, $\left.v \mathrm{~cm}^{-1}\right): 2934(\mathrm{C}-\mathrm{H}), 1754(\mathrm{C}=\mathrm{O}), 1594$ $(\mathrm{HC}=\mathrm{N}), 1372\left(\mathrm{CH}_{3}\right), 1042,1228(\mathrm{C}-\mathrm{O}),{ }^{1} \mathrm{H}$ NMR $(300$ $\mathrm{MHz}_{\mathrm{CDCl}}$ ): $\delta 1.92$ (s, 3H, OAc), 2.03 (s, 3H, OAc), $2.06(\mathrm{~s}, 3 \mathrm{H}, \mathrm{OAc}), 2.08(\mathrm{~s}, 3 \mathrm{H}, \mathrm{OAc}), 1.35(\mathrm{t}, J=7.2 \mathrm{~Hz}, 3 \mathrm{H}$, $\left.\mathrm{CH}_{3}\right), 2.83-2.87\left(\mathrm{~m}, 2 \mathrm{H}, \mathrm{CH}_{2}\right) 3.97-3.99(\mathrm{~m}, 1 \mathrm{H}, \mathrm{H}-6 \mathrm{a})$, $4.16(\mathrm{~d}, J=12 \mathrm{~Hz}, 1 \mathrm{H}, \mathrm{H}-6 \mathrm{~b}), 4.28-4.33(\mathrm{~m}, 1 \mathrm{H}, \mathrm{H}-5)$, $5.26(\mathrm{t}, J=9.6 \mathrm{~Hz}, 1 \mathrm{H}, \mathrm{H}-4), 5.41(\mathrm{t}, J=9.6 \mathrm{~Hz}, 1 \mathrm{H}, \mathrm{H}-2)$, $5.79(\mathrm{t}, J=9 \mathrm{~Hz}, 1 \mathrm{H}, \mathrm{H}-3), 6.17(\mathrm{~d}, J=9.3 \mathrm{~Hz}, 1 \mathrm{H}, \mathrm{H}-1), 7.69$ (d, $J=4.5 \mathrm{~Hz}, 2 \mathrm{H}$ pyridine), 8.77 (d, $J=3.9 \mathrm{~Hz}, 2 \mathrm{H}$ pyridine) $10.81(\mathrm{~s}, 1 \mathrm{H}, \mathrm{HC}=\mathrm{N}),{ }^{13} \mathrm{C}-\mathrm{NMR}\left(75 \mathrm{MHz}, \mathrm{CDCl}_{3}\right)$ : $\delta(\mathrm{PPM}), 10.11,18.92,20.60,61.64,67.71,68.98,73.65$, $74.50,81.37,121.86,140.03,150.64,153.15,155.90$, 164.11, 168.76, 169.40, 170.20, 170.66. Calcd: C, 51.15; H, 5.19; N, 12.43; S, 5.69\%; Found: C, 51.01; H, 5.21; N, 12.53 ; S, 5.73\%. 
Synthesis of 4-(pyridine-3-yl methylene-amino)-5-ethyl-2-yl-3-(2,3,4,6-tetra-O-acetyl- $\beta$-D-gluco pyranosyl Sulfonyl) -1,2,4-triazole (7e):

White crystals, $78 \%(0.43 \mathrm{~g})$, m.p: $105-107^{\circ} \mathrm{C}$, FT-IR $\left(\mathrm{KBr}, \mathrm{v} \mathrm{cm}^{-1}\right): 2947(\mathrm{C}-\mathrm{H}), 1753(\mathrm{C}=\mathrm{O}), 1591(\mathrm{HC}=\mathrm{N})$, $1371\left(\mathrm{CH}_{3}\right), 1042,1229(\mathrm{C}-\mathrm{O}),{ }^{1} \mathrm{H}$ NMR $(300 \mathrm{MHz}$, $\mathrm{CDCl}_{3}$ ): $\delta 1.92(\mathrm{~s}, 3 \mathrm{H}, \mathrm{OAc}), 2.03$ (s, 3H, OAc), $2.06(\mathrm{~s}, 3 \mathrm{H}$, $\mathrm{OAc}), 2.08$ (s, 3H, OAc), $1.34\left(\mathrm{t}, J=7.2 \mathrm{~Hz}, 3 \mathrm{H}, \mathrm{CH}_{3}\right), 2.79-$ $2.88\left(\mathrm{~m}, 2 \mathrm{H}, \mathrm{CH}_{2}\right), 3.97-4.01(\mathrm{~m}, 1 \mathrm{H}, \mathrm{H}-6 \mathrm{a}), 4.17(\mathrm{~d}$, $J=12.6,1 \mathrm{H}, \mathrm{H}-6 \mathrm{~b}), 4.28-4.33$ (m, 1H, H-5), 5.26 (t, J=9.9 $\mathrm{Hz}, 1 \mathrm{H}, \mathrm{H}-4), 5.41(\mathrm{t}, J=9.3 \mathrm{~Hz}, 1 \mathrm{H}, \mathrm{H}-2), 5.79(\mathrm{t}, J=9.3 \mathrm{~Hz}$, $1 \mathrm{H}, \mathrm{H}-3), 6.18$ (d, J=9.3 Hz, 1H, H-1),7.41-7.45 (m, 1H, pyridine), 8.18 (d, $J=7.8 \mathrm{~Hz}, 1 \mathrm{H}$ pyridine) 8.75 (d, $J=4.5$ $\mathrm{Hz}, 1 \mathrm{H}$ pyridine) $9.01(\mathrm{~s}, 1 \mathrm{H}$, pyridine $) 10.69(\mathrm{~s}, 1 \mathrm{H}$, $\mathrm{HC}=\mathrm{N}) .{ }^{13} \mathrm{C}-\mathrm{NMR}\left(75 \mathrm{MHz}, \mathrm{CDCl}_{3}\right): \delta(\mathrm{PPM}), 10.11$, $18.93,20.60,61.65,67.71,68.99,73.66,74.46,81.44$, $123.92,128.70,134.79,149.81,153.05,158.26,164.02$, 168.76, 169.40, 170.18, 170.66. Calcd: C, 51.15; H, 5.19; N, 12.43; S, 5.69\%; Found: C, 51.07; H, 5.21; N, 12.60; S, $5.61 \%$.

Synthesis of 4-(pyridine-2-yl methylene-amino) -5-ethyl-2-yl-3- (2,3,4,6-tetraO-acetyl- $\beta$-D-gluco pyranosyl Sulfonyl) -1,2,4-triazole (7f):

yellow crystals $79 \%(0.87 \mathrm{~g}), \mathrm{m} . \mathrm{p}: 55-57{ }^{\circ} \mathrm{C}$; FT-IR $\left(\mathrm{KBr}, \mathrm{v} \mathrm{cm}^{-1}\right): 2974(\mathrm{C}-\mathrm{H}), 1753(\mathrm{C}=\mathrm{O}), 1586(\mathrm{HC}=\mathrm{N})$, $1372\left(\mathrm{CH}_{3}\right), 1044,1229$ (C-O). ${ }^{1} \mathrm{H}$ NMR $(300 \mathrm{MHz}$, $\left.\mathrm{CDCl}_{3}\right): \delta 1.92(\mathrm{~s}, 3 \mathrm{H}, \mathrm{OAc}), 2.02$ (s, 3H, OAc), 2.06 (s, 3H, OAc), 2.08 (s, 3H, OAc), $1.33\left(\mathrm{t}, J=7.2 \mathrm{~Hz}, 3 \mathrm{H}, \mathrm{CH}_{3}\right), 2.77-$ $2.89\left(\mathrm{~m}, 2 \mathrm{H}, \mathrm{CH}_{2}\right), 3.96-4(\mathrm{~m}, 1 \mathrm{H}, \mathrm{H}-6 \mathrm{a}), 4.16(\mathrm{~d}, J=11.4$ $\mathrm{Hz}, 1 \mathrm{H}, \mathrm{H}-6 \mathrm{~b}), 4.27-4.33$ (m, 1H, H-5), 5.25 (t, $J=9.6 \mathrm{~Hz}$, $1 \mathrm{H}, \mathrm{H}-4), 5.40(\mathrm{t}, J=9.3 \mathrm{~Hz}, 1 \mathrm{H}, \mathrm{H}-2), 5.77(\mathrm{t}, J=9.3 \mathrm{~Hz}, 1 \mathrm{H}$, $\mathrm{H}-3), 6.19$ (d, J=9.3 Hz, 1H, H-1), 7.39-7.43 (m,1H, pyridine) $7.81(\mathrm{t}, J=7.5 \mathrm{~Hz}, 1 \mathrm{H}$, pyridine) $8.07(\mathrm{~d}, J=7.8 \mathrm{~Hz}, 1 \mathrm{H}$, pyridine) 8.73 (d, J=3.9 Hz, 1H, Pyridin), 10.61 (s, $1 \mathrm{H}$, $\mathrm{CH}=\mathrm{N}) .{ }^{13} \mathrm{C}-\mathrm{NMR}\left(75 \mathrm{MHz}, \mathrm{CDCl}_{3}\right): \delta(\mathrm{PPM}), 10.10$, $18.92,20.56,61.70,67.82,69.09,73.68,74.48,81.52$, $121.79,125.84,136.70,150.18,152.05,152.95,162.33$, 164.27, 168.76, 169.38, 170.11, 170.63. Calcd: C, 51.15; H, 5.19; N, 12.43; S, 5.69\%; Found: C, 51.25; H, 5.28; N, 12.33; S, $5.72 \%$.

Synthesis of 4-(pyridine-3-yl methylene-amino) -5-propyl-2-yl-3- $(2,3,4,6$-tetra-O-acetyl- $\beta$-D-gluco pyranosyl Sulfonyl) -1,2,4-triazole (7g):

White crystals; $81 \%$ (0.93 g), m.p: $63-65^{\circ} \mathrm{C}$; FT-IR $\left(\mathrm{KBr}, \mathrm{v} \mathrm{cm}^{-1}\right): 2962(\mathrm{C}-\mathrm{H}), 1754(\mathrm{C}=\mathrm{O}), 1590(\mathrm{HC}=\mathrm{N})$, $1369\left(\mathrm{CH}_{3}\right), 1048,1229$ (C-O). ${ }^{1} \mathrm{H}$ NMR $(300 \mathrm{MHz}$, $\left.\mathrm{CDCl}_{3}\right): \delta 1.92(\mathrm{~s}, 3 \mathrm{H}, \mathrm{OAc}), 2.03$ (s, 3H, OAc), 2.06 (s, 3H, $\mathrm{OAc}$ ), 2.08 (s, 3H, OAc), 1.01 (t, J=7.5Hz, 3H, $\left.\mathrm{CH}_{3}\right), 1.74-$ $1.86\left(\mathrm{~m}, 2 \mathrm{H}, \mathrm{CH}_{2}\right) 2.73-2.86\left(\mathrm{~m}, 2 \mathrm{H}, \mathrm{CH}_{2}\right) 3.97-3.99(\mathrm{~m}$, $1 \mathrm{H}, \mathrm{H}-6 \mathrm{a}), 4.13-4.19(\mathrm{~m}, 1 \mathrm{H}, \mathrm{H}-6 \mathrm{~b}), 4.27-4.32(\mathrm{~m}, 1 \mathrm{H}$, $\mathrm{H}-5), 5.25$ (t, $J=9.6 \mathrm{~Hz}, 1 \mathrm{H}, \mathrm{H}-4), 5.41(\mathrm{t}, J=9.3 \mathrm{~Hz}, 1 \mathrm{H}$, $\mathrm{H}-2), 5.79(\mathrm{t}, J=9.3 \mathrm{~Hz}, 1 \mathrm{H}, \mathrm{H}-3), 6.18(\mathrm{~d}, J=9.6 \mathrm{~Hz}, 1 \mathrm{H}$, $\mathrm{H}-1), 7.49-7.53(\mathrm{~m}, 1 \mathrm{H}$, pyridine), $8.25(\mathrm{~d}, J=7.8 \mathrm{~Hz}, 1 \mathrm{H}$ pyridine) $8.77(\mathrm{~d}, J=3.6 \mathrm{~Hz}, 1 \mathrm{H}$ pyridine $) 9.04(\mathrm{~s}, 1 \mathrm{H}$, pyr- idine) $10.78(\mathrm{~s}, 1 \mathrm{H}, \mathrm{HC}=\mathrm{N}):{ }^{13} \mathrm{C}-\mathrm{NMR}\left(75 \mathrm{MHz}, \mathrm{CDCl}_{3}\right)$ : $\delta(\mathrm{PPM}), 13.49,19.22,20.73,26.93,61.66,67.78,69,73.69$, $74.51,81.45,124.35,129.37,135.99,151.91,155.24,157.16$, $162.33,163.99,168.69,169.35,170.14,170.60$. Calcd: C, 51.99; H, 5.41; N, 12.12; S, 5.55\%; Found: C, 51.89; H, 5.50; N, 12.23 ; S, 5.42\%.

\section{Conclusions}

In summary, a series of 3 -S- $\beta$-D-glucosides-4arylideneamino-5-alkyl-1,2,4-triazoles $7(\mathbf{a}-\mathbf{e})$ was prepared by reaction of 4-amino-5-alkyl-4H-1,2,4-triazole-3thiol Schiff bases $\mathbf{5}(\mathbf{a}-\mathbf{f})$ and 2,3, 4,6-tetra-O-acetyl- $\alpha$-D-glucopyranosyl bromide (6) in presence of potassium carbonate at room temperature for $24 \mathrm{~h}$ and then $3 \mathrm{~h}$ reflux. The structures of the target compounds have been characterized by ${ }^{1} \mathrm{H} \mathrm{NMR},{ }^{13} \mathrm{C}$ NMR, and FTIR. All the newly synthesized compounds have been evaluated for their antimicrobial activities in vitro against two gram $(+)$ bacteria, two gram (-) bacteria and two fungi. These compounds exhibited better antifungal activity than antibacterial activity. Though compound $(7 \mathbf{b})$ was found to have the highest activity against Bacillus cereus and compound (7a) was found to have the highest activity against Candida albicans among all the tested compounds.

\section{Acknowledgments}

The authors are grateful to Urmia University for providing a fellowship for the present work.

\section{References}

1. H. A. Burch, W. O. Smith, J. Med. Chem. 1966, 9, 405-408. DOI:10.1021/jm00321a033

2. A. Foroumadi, S. Mansouri, Z. Kiani, A. Rahmani, Eur. J. Med. Chem. 2003, 38, 851-854.

DOI:10.1016/S0223-5234(03)00148-X

3. L. P. Guan, Z. S. Quan, Mini Rev. Med. Chem. 2016, 16, 323342. DOI:10.2174/1389557515666150101100909

4. M. Ziaie, K. A. Dilmaghani, A. Tukmechi, Acta Chimica Slovenica. 2017, 64, 895-901. DOI:10.17344/acsi.2017.3506

5. A. Srinivas, M. Sunitha, P. Karthik, K. V. Reddy, Acta Chimica Slovenica. 2017, 64, 1030-1041.

DOI:10.17344/acsi.2017.3805

6. X. Collin, A. Sauleau, J. Coulon, Bioorg. Med. Chem. Lett. 2003, 1ff3, 2601-2605.

7. Z. Rezaeia, S. Khabnadideha, K. Pakshirb, Z. Hossainia, F. Amiria, E. Assadpour, European Journal of Medicinal Chemistry. 2009, 44, 3064-3067.

DOI:10.1016/j.ejmech.2008.07.012

8. G. Tanaka, Japan Kokai. 1974, 973, 7495. Chem. Abstr. 1975, $82,156320 \mathrm{~h}$. 
9. M. Kalhor, M. Shabani, I. Nikokar, SR. Banisaeed, Iran. J. Pharm. Res. 2015, 14, 67-75.

10. M. Amir, K. Shikha, Eur. J. Med. Chem. 2004, 39, 535-545. DOI:10.1016/j.ejmech.2004.02.008

11. S. Botros, O. M. Khalil, M. M. Kamel, Y. S. El-Dash, Acta Chimica Slovenica. 2017, 64, 102-116.

DOI:10.17344/acsi.2016.2901

12. S. A. Abdel-Aziz, H. A. Allimony, H. M. El-Shaaer, Phosphorus Sulfur Silicon Rel. Elem. 1996, 67, 113.

13. T. K. Venkatachalam, E. A. Sudbeck, C. Mao, M. F. Uckun, Med. Chem. Lett, 2001, 11, 523-528.

DOI:10.1016/S0960-894X(01)00011-7

14. M. Y. Mhasalkar, M. H. Shah, S. T. Nikam, K. G. Anantanarayanan, C. V. Deliwala, Journal of Medicinal Chemistry. 1970, 13, 672-674. DOI:10.1021/jm00298a021

15. S. Rollas, N. Kalyoncuoglu, D. Sur-Altiner, Y. Yegenoglu, Pharmazie. 1993, 48, 308-309.

16. S. Giri, H. Singh, L.D.S. Yadav, R. K. Kahre, J. Indian Chem. Soc. 1978, 55, 168-171.

17. D. J. Sheehan, C. A. Hitchcock, C. M. Sibley, Clin. Microbiol. Rev.1999, 12, 40-79. DOI:10.1128/CMR.12.1.40

18. K. M. Crofton, V.M. Boncek, L.W. Reiter,-Fundam Appl Toxicol. 1988, 10, 459-465. DOI:10.1016/0272-0590(88)90292-8

19. K. Akbari Dilmaghani, N. H. Jazani, F. Nasuhi Pur, N. Shokoufeh F. Ghadiri, M. Fakhraee. Chemistry of Heterocyclic Compounds. 2012, 48, 362-367.

20. Deepa Gupta, D. K. Jain, J. Adv. Pharm. Technol. Res. 2015, 6, 145-146.

21. SUN. Xiao-Hong, TAO. Yan, LIU. Yuan-Fam, CHEN. Bang, JIA. Ying-Qi, YANG. Jian-Wu, Chinese Journal of Chemistry. 2008, 26, 1133-1136.

22. K. Akbari Dilmaghani, F. Nasuhi Pur, M. Hatami Nezhad, Iran J Pharm Res. 2015, 14, 693-699.

23. M. G. Dhapalapur, S.S. Sabnis. C.V. Deliwala, J. Med. Chem. 1968, 11, 1014-1019. DOI:10.1021/jm00311a022

24. S. A. M. K. Nasser, Carbohydr. Res. 2006, 341, 2187-2199. DOI:10.1016/j.carres.2006.06.007

25. Weal A. El-Sayed, Randa E. Abdel Megeid, Hebat-Allah S. Abbas, Arch Pharm Res. 2011, 34, 1085-1096.

DOI:10.1007/s12272-011-0706-y
26. D. Ji, J. Lu, B. Lu, C. Xin, J. Mu, J. Li, C. Peng, X. Bao, Bioorganic \& Medicinal Chemistry Letters. 2013, 23, 1997-2000. DOI:10.1016/j.bmcl.2013.02.038

27. K. Akbari Dilmaghani, F. Nasuhi Pur, N. H. Jazani, A. Alavi, Z. Niknam, F. Mirfakhraee, Phosphorus Sulfur Silicon. 2014, 189, 81-87. DOI:10.1080/10426507.2013.789877

28. K. Akbari Dilmaghani, F. Nasuhi Pur, M. Mahammad pour, J. Mahammad nejad, Iranian Journal of Pharmaceutical Research. 2016, 15, 777-782.

29. NCCLS (National Committee for Clinical Laboratory Standards) Performance standards for antimicrobial disk susceptibility test ( $6^{\text {th }}$ ed.). Wayne, PA: Approved Standard. M2-A6. 1997, 35.

30. M. B. Bahadori, H. Valizadeh, B. Asghari, L. Dinparast, M. Moridi Farimani, S. Bahadori, Journal of Functional Foods. 2015, 18, 727-736. DOI:10.1016/j.jff.2015.09.011

31. M. B. Bahadori, B. Asghari, L. Dinparast, G. Zengin, C. Sarikurkcu, M. A. Mohammadi, Sh. Bahadori, LWT - Food Science and Technology. 2017, 75, 42-45.

DOI:10.1016/j.lwt.2016.08.048

32. H. Beyer, C.F. Kroger, Liebigs Ann. Chem. 1960, 637, 135. DOI:10.1002/jlac.19606370111

33. K. S. Ashok, R.N. Singh, R. N. Handa, S. N. Dubey, P. J. Squattrito, J. Mol. Struct. 1998, 470, 61-69. DOI:10.1016/S0022-2860(98)00470-0

34. C. Guimon, G. Pfister-Guillouzo, Tetrahedron. 1974, 30, 3831-3838. DOI:10.1016/S0040-4020(01)97072-3

35. F. Malbec, R. Milcent, G. Barbier, J. Heterocycl. Chem. 1984, 21, 1689-1698. DOI:10.1002/jhet.5570210624

36. V. I. Kelarev, G. A. Shvekhgeimer, A. F. Lunin, Khim. Geterotsikl. Soedin. 1984, 1271-1276.

37. S. Gopinathan, S. A. Pardhy, A. P. Budhkar, C. Gopinathan Synth. React. Inorg. Metal. Org. Chem. 1988, 18, 823-836. DOI:10.1080/00945718808060824

38. D. D. Perrin, W. L. F. Armarego, Purification of Laboratory Chemicals, Pergamon Press, Oxford. 1988.

39. Vogel, I. Arthur, Vogel's Textbook of Practical Organic Chemistry. $1989,5^{\text {th }}$ ed.

\section{Povzetek}

$\mathrm{Z}$ reakcijami med 4-amino-5-alkil-4H-1,2,4-triazol-3-tiolnimi Schiffovimi bazami in 2,3,4,6-tetra-O-acetil- $\alpha$-D-glukopiranozil bromidom smo sintetizirali novo serijo 3-S- $\beta$-D-glukozid-4-arilidenamino-5-alkil-1,2,4-triazolov. Strukture pripravljenih spojin smo določili $z{ }^{1} \mathrm{H}$ NMR, ${ }^{13} \mathrm{C}$ NMR, FT-IR in elementno mikroanalizo. Za vse novopripravljene spojine smo in vitro določili antibakterijsko in protiglivično delovanje na dve Gram pozitivni bakteriji (Bacillus cereus (PTCC 1015) in Staphylococcus aureus (ATCC 25923)), dve Gram negativni bakteriji (Pseudomonas aeruginosa (ATCC 27853) in Escherichia coli (PTCC 1399)) ter na dve glivi (Aspergillus niger (PTCC 5012) in Candida albicans (PTCC 5027)). 